\title{
ENSAIO TEÓRICO
}

DOI: 10.18310/2358-8306.v6n12.a9

\author{
IDENTIFICAÇÃO PRECOCE, INTERVENÇÃO TERAPÊUTICA E \\ INSERÇÃO ESCOLAR NA CRIANÇA DEFICIENTE VISUAL
}

\author{
EARLY IDENTIFICATION, THERAPEUTIC INTERVENTION, AND \\ SCHOOL INSERTION FOR VISUALLY DISABLED CHILDREN
}

Paula Berteli Pelizaro (Orcid: 0000-0001-5313-8968) ${ }^{1}$

Patricia Ribeiro Marcacine (Orcid: 0000-0003-1784-2231)

Suraya Gomes Novais Shimano (Orcid: 0000-0002-9421-9227)

Karina Pereira (Orcid: 0000-0001-7486-1004) $)^{1,3}$

Nuno Miguel Lopes Oliveira (Orcid: 0000-0001-7295-7491) ) $^{1,3}$

Jessica Carvalho Lima (Orcid: 0000-0003-0972-1886)

Isabel Aparecida Porcatti de Walsh (Orcid: 0000-0002-2317-1326) (1,3 $^{1}$

Contato

Isabel Aparecida Porcatti de Walsh

E-mail: isabelpwalsh@gmail.com

${ }^{1}$ Programa de Pós-Graduação em Fisioterapia, Universidade Federal do Triângulo Mineiro/Universidade Federal de Uberlândia, Minas Gerais, Brasil. ${ }^{2}$ Programa de Pós-Graduação em Atenção à Saúde, Universidade Federal do Triângulo Mineiro, Minas Gerais, Brasil.

${ }^{3}$ Departamento de Fisioterapia Aplicada, Universidade Federal do Triângulo Mineiro, Minas Gerais, Brasil.

\section{RESUMO}

A Política Nacional de Atenção Integral à Saúde da Criança contempla eixos estratégicos que visam à qualificação e à orientação das ações e serviços de saúde que são desenvolvidos em todo o território nacional. Dentre seus eixos, destaca-se o que abrange a atenção à saúde de crianças com deficiência ou em situações específicas e de vulnerabilidade. Por ser uma política ampla, torna-se importante refletir sobre a criança com deficiência visual. Os conceitos, dados epidemiológicos, avaliação, direitos e diretrizes da deficiência visual são pouco conhecidos e divulgados, considerando que há uma lacuna de conhecimento para os profissionais da área da saúde e da educação referente a essa temática. A melhor forma de realizar a prevenção e a promoção de saúde nessas crianças é por meio da capacitação eficiente dos profissionais para realizar avaliações e diagnósticos precoces, com a finalidade de encaminhá-las para serviços especializados, tornando, assim, as ações efetivas e visando à autonomia e à qualidade de vida dessa população.

Palavras-chave: Transtornos da visão; Criança; Inclusão educacional; Autonomia pessoal.

\begin{abstract}
The National Policy for Comprehensive Child Health Care includes strategic axes aimed at qualifying and guiding health actions and services that are developed throughout the national territory. Among its axes, we highlight what covers health care for children with disabilities or in specific and vulnerable situations. As it is a broad policy, it is important to reflect on the visually impaired children. The concepts, epidemiological data, assessment, rights, and guidelines of visual impairment are poorly known and disseminated, considering that there is a knowledge gap for health and education professionals regarding this topic. The best way to carry out prevention and health promotion among those children is through the efficient training of professionals to perform early assessments and diagnoses, with the purpose of referring them to specialized services, thus making the actions effective and aiming at the autonomy and quality of life of this population.

Keywords: Visual disorders; Kid; Mainstreaming (Education); Personal Autonomy.
\end{abstract}


POLÍTICA NACIONAL DE ATENÇÃO INTEGRAL À SAÚDE DA CRIANÇA (PNAISC) E CRIANÇAS COM DEFICIÊNCIA VISUAL

A Política Nacional de Atenção Integral à Saúde da Criança (Pnaisc), instituída no âmbito do Sistema Único de Saúde (SUS), tem como objetivos proteger e promover o aleitamento materno e a saúde da criança, oferecer atenção e cuidados integrais e integrados, reduzir a morbimortalidade e proporcionar um ambiente facilitador à vida com condições de desenvolvimento adequado e digno de existência. Entre seus eixos estratégicos, destaca-se o VI do art. $6^{\circ}$ que trata da Atenção à saúde de crianças com deficiência ou em situações específicas e de vulnerabilidade ${ }^{1}$.

A deficiência visual é classificada por meio da avaliação da acuidade e/ou campo visual e em categorias que incluem desde a ausência total da visão até a perda visual leve $e^{2}$. A baixa visão pode ser considerada a acuidade visual corrigida no melhor olho, sendo menor que 0,3 e maior ou igual a 0,05 ou campo visual menor que 20 graus no melhor olho com correção óptica, e considera-se cegueira quando a acuidade visual for inferior a $0,05^{2,3}$.

A Organização Mundial da Saúde estima que, no Brasil, 19 milhões de crianças apresentem alguma deficiência visual, sendo 17,5 milhões com baixa visão, e 1,5 milhão cega ${ }^{4}$. Estudos destacam que a baixa visão está relacionada com glaucoma congênito, toxoplasmose, catarata infantil, deficiência visual de origem cortical, alterações no nervo óptico e retinopatia da prematuridade ${ }^{2,5-7}$. Ainda, desde 2016, o Zika vírus, transmitido pelo mosquito Aedes Aegypti, tem sido o responsável em ocasionar danos cerebrais de extrema gravidade, malformações e alterações oculares graves ${ }^{8-9}$.

Segundo a Agência Internacional para a Prevenção da Cegueira (IAPB), no Brasil, há cerca de 29 mil crianças cegas devido a doenças oculares que poderiam ter sido evitadas ou tratadas precocemente $^{10}$. Estima-se que cerca de $50 \%$ a $80 \%$ das causas são preveníeis (toxoplasmose, deficiência de vitamina A) ou tratáveis (retinopatia da prematuridade, catarata, glaucoma $)^{2,11,12}$. Além disso, estima-se que 12,8 milhões de crianças entre 5 anos e 15 anos apresentem deficiência visual por erros refrativos não corrigidos, sendo estes identificados como problema de saúde pública em crianças, constituindo-se a principal causa de deficiência visual em escolares $^{13-15}$.

Dessa maneira, ressalta-se a importância de conhecer os conceitos e fortalecer as diretrizes e direitos das crianças com deficiência visual, visando às melhores formas de prevenção, identificação, diagnóstico, tratamento precoce e habilitação visual das doenças que acometem o sistema ocular, garantindo melhores chances de desempenho dessa população.

\section{IDENTIFICAÇÃO DE POSSÍ- VEIS ALTERAÇÕES OCULA- RES E ORIENTAÇÕES ÀS EQUI- PES MULTIPROFISSIONAIS}

A identificação de possíveis alterações oculares se inicia logo após o nascimento, por meio do rastreamento visual ativo, realizando a inspeção externa e o 
teste do reflexo vermelho. Quando se detectam a doença ocular ou alguns fatores de risco, o neonato deve ser encaminhado imediatamente para o serviço oftalmológico especializado ${ }^{2}$.

Com o objetivo de oferecer orientações às equipes multiprofissionais para o cuidado da saúde ocular da criança e identificação dos fatores de risco abrangendo o pré-natal, neonatal, até o final da infância, a Secretaria de Atenção à Saúde (SAS) do Ministério da Saúde (MS), com colaboração da Área Técnica Saúde da Criança e Aleitamento Materno/Dapes/ SAS/MS, do Conselho Brasileiro de Oftalmologia ( $\mathrm{CBO}$ ), da Sociedade Brasileira de Oftalmologia Pediátrica (SBOP) e da Sociedade Brasileira de Pediatria (SBP), elaborou as Diretrizes de Atenção à Saúde Ocular na Infância: detecção e intervenção precoce para a prevenção de deficiências visuais, que consiste na utilização de procedimentos simples e rápidos, a fim de que se possa detectar, ainda no neonato, uma possível alteração visual, iniciando o processo de reabilitação da criança ${ }^{16}$.

Qualquer suspeita de alterações visuais deve ser encaminhada ao serviço especializado em oftalmologia para a realização de exame ocular geral, que consiste em exame externo da face e olhos, avaliação dos reflexos fotomotores das pupilas, avaliação da motilidade ocular e medida da acuidade visual ${ }^{17}$.
A Portaria $\mathrm{n}^{\circ} 3.128$ estabelece que as Redes Estaduais de Atenção à Pessoa com Deficiência Visual sejam compostas por ações na atenção básica e Serviços de Reabilitação Visual ${ }^{19}$; e a lei brasileira de inclusão explicita que é dever do estado assegurar à pessoa com deficiência o direito à saúde por meio de uma atenção integral em todos os níveis de complexidade, por intermédio do SUS, garantindo o acesso universal e igualitário e promovendo o bem-estar pessoal, social e econômico ${ }^{18}$.

A avaliação das funções visuais (funcionamento das estruturas oculares: acuidade visual, campo visual, adaptação visual, visão binocular, sensibilidade aos contrastes, visão de cores, entre outras, geralmente avaliadas por oftalmologistas com testes e exames clínicos específicos) e da visão funcional (descrição de como a pessoa funciona ou é eficiente para a realização de atividades cotidianas relacionadas à visão: contato de olho, fixar e seguir um objeto em movimento, discriminação e reconhecimento de formas, tamanhos e cores etc.) fornecem aos profissionais, tanto da saúde quanto os da educação, subsídios fundamentais para o trabalho de habilitação/reabilitação visual ${ }^{5,19,20}$.

$\mathrm{Na}$ idade escolar, estima-se que $10 \%$ das crianças apresentem alguma perturbação oftalmológica (erros de refração, estrabismo, ambliopia, sequela de acidente ocular, malformações congênitas, entre 
outros $)^{21}$. Para garantir o respeito às diversidades apresentadas por elas, assegurar e promover a plena realização de todos os seus direitos, deve ser considerado que a baixa visão pode limitar o número de experiências e informações, interferindo no desenvolvimento motor, cognitivo e emocional. É importante ponderar que, por mais que a visão seja comprometida, os escolares devem ser estimulados a usar seu resíduo visual, que por sua vez, pode ser melhorado e potencializado por meio da utilização de recursos de tecnologia assistiva. As escolas devem estar preparadas para receber os escolares com baixa visão, pois é direito deles frequentar escolas inclusivas e que atendam às suas necessidades, assim como o atendimento em instituições especializadas ${ }^{22}$.

A Lei de Diretrizes e Bases da Educação Nacional incorpora a noção de que o sistema educacional deve estar centrado nos alunos, quaisquer que sejam as suas características. Regulamenta a Educação Especial como uma modalidade de ensino que deve realizar-se, preferencialmente, na rede regular de ensino, estabelece diretriz e base da educação nacional e prevê serviços de apoio especializado na escola regular, para atender às peculiaridades das pessoas com deficiência ${ }^{23}$.

Atualmente, na perspectiva educacional, o uso do resíduo visual é o que define a baixa visão, ou seja, o critério de funcionalidade. Busca-se a caracterização do "como" a pessoa enxerga e não apenas o "quanto" ela enxerga ${ }^{24}$. Essa mudança de paradigma trouxe novas possibilidades à educação das crianças com baixa visão, sendo que os programas de educação, habilitação, reabilitação passaram a considerar tal premissa. Esses escolares ne- cessitam de um programa de intervenção amplo que englobe os aspectos clínicos, reabilitacionais e educacionais, com ênfase no uso máximo do resíduo visual, por meio da realização de atividades específicas que estimulem essa visão, atendendo às particularidades dessas crianças ${ }^{25,26}$.

Ainda, destacam-se a Portaria $\mathrm{n}^{\circ}$ 319/99, que traz diretrizes para a adoção de normas para o uso, ensino, produção e difusão do sistema braile ${ }^{27}$, e a Lei ${ }^{\circ}$ 10.753/03, que institui a Política Nacional do Livro, assegurando o direito de pessoas com deficiência visual à leitura ${ }^{28}$. O Decreto $n^{\circ}$ 5.296/04 estabelece que o Poder Público adote mecanismos para incentivar a disponibilização, por intermédio de meio magnético, em formato de texto, das obras públicas do país ${ }^{29}$; e o Decreto $\mathrm{n}^{\circ}$ 7.611/11 coloca que o Ministério da Educação (MEC) prestará apoio financeiro e técnico para a produção e distribuição de recursos de acessibilidade educacionais, entre os quais estão incluídos livros didá$\operatorname{ticos}^{30}$.

Considerando que as dificuldades mais comuns apresentadas pelos escolares com baixa visão, na realização de suas atividades acadêmicas, estão relacionadas com o uso da lousa e com as atividades de perto, como a leitura e a escrita, estas podem ser minimizadas com o uso de recursos de tecnologia assistiva ${ }^{31}$, ou seja, quaisquer soluções que promovam o melhor desempenho em suas atividades cotidianas, com a finalidade de eliminar ou minimizar as dificuldades visuais.

Tais recursos podem ser de alta ou baixa tecnologia e classificados como ópticos (lentes de contato, sistemas telescópios manuais de foco ajustável e siste- 
mas telescópios de foco fixo, montados em armação, lupas manuais, fixas, de apoio, óculos comuns e óculos especiais com lentes de grande aumento), não ópticos (modificam os materiais e os ambientes para promoverem o melhor desempenho visual e podem ser para ampliação dos objetos, para posicionamento e postura, para escrita e leitura e para controle de iluminação, como ampliação de livros didáticos, das pautas dos cadernos, agenda escolar, da iluminação adequada, de acessórios como o suporte para leitura e escrita, do aumento do contraste por meio de lápis com grafite mais forte, canetas hidrográficas, uso de cores bem contrastantes como a tinta preta em papel branco e giz branco ou amarelo para aumentar o contraste com o fundo da lousa) e eletrônicos e de informática (computadores utilizando-se o sistema ampliado, o sistema sonoro, ou sem nenhuma adaptação) ${ }^{22,32-34}$.

A Nota Técnica n ${ }^{\circ} 05 / 011$ coloca os aspectos legais e técnicos relacionados com a utilização do Mecdaisy, software direcionado à leitura de livros por intermédio de meio magnético ${ }^{35}$. Além do mais, o Centro de Apoio Pedagógico para Atendimento às Pessoas com Deficiência Visual (CAP), projeto da Secretaria de Educação Especial (SEESP) do MEC, tem como objetivo garantir aos deficientes visuais o acesso a um ensino de qualidade, por meio de materiais de apoio pedagógico e suplementação didática. Qualquer estudante cego ou com visão subnormal pode utilizar os serviços do CAP, mas é dada prioridade a quem está matriculado no ensino fundamental de escolas públicas. Entre os serviços prestados pelas unidades, encontram-se a capacitação de recursos humanos e a oferta de estágios aos profissionais da área e alunos de instituições especializadas. Três núcleos integram a sua estrutura: de produção Braille, que auxilia o MEC na impressão e distribuição de livros didáticos para deficientes visuais matriculados no ensino fundamental; de Apoio Pedagógico; de Tecnologia e de Convivência ${ }^{36}$.

Quanto à parceria entre a saúde e a educação, desde o ano de 2007, o Brasil dispõe do Programa de Saúde na escola, cuja proposta indica que professores, capacitados por profissionais de saúde, possam detectar situações-problema como dificuldades visuais e fazer o encaminhamento dos alunos aos órgãos de saúde ${ }^{37}$.

\section{CONSIDERAÇÕES FINAIS}

Para que as informações e a efetividade das políticas e diretrizes propostas para crianças com deficiência visual sejam eficazes, deverá ocorrer atuação de equipes multidisciplinares desde a atenção básica até aos atendimentos em serviços especializados, divulgando as informações para a população, fortalecendo os projetos e programas existentes e promovendo novas ideias para abranger essa po- 
pulação de forma adequada. Além disso, devem-se conhecer as melhores formas de avaliar e estimular essas crianças, promovendo estudos que enfatizem suas particularidades para, assim, adquirir os conhecimentos necessários para orientar os pais e/ou cuidadores e profissionais sobre a melhor forma de estimular as crianças com deficiência visual, buscando maior independência e qualidade de vida dessa população.

Nesse sentido, promover o desempenho funcional à criança com deficiência visual pode ser definido como um dos objetivos a serem alcançados pelo fisioterapeuta, uma vez que o processo de reabilitação envolve condutas com foco na redução das perdas funcionais, promoção da saúde, valorização da autonomia e independência ${ }^{38,39}$.

Embora existam leis, programas e diretrizes, nota-se a falta de informação, de dados epidemiológicos e de divulgação dessa temática. Diante dos fatores apresentados, é essencial que os profissionais da área da saúde sejam capacitados de forma eficiente para que consigam identificar a deficiência visual, efetivando o diagnóstico precoce, as formas de tratamento e para que sejam capazes de realizar o encaminhamento dessas crianças para a intervenção precoce; além disso, é essencial que divulguem tais informações para a população.

\section{REFERÊNCIAS}

1. Brasil. Portaria $\mathrm{n}^{\circ} 1.130$, de 05 de agosto de 2015. Institui a Política Nacional de Atenção Integral à Saúde da Criança (PNAISC) no âmbito do Sistema Único de Saúde (SUS). Diário Oficial da União. 2015; 05 ago.

2. Brasil. Ministério da Saúde. Secretaria de atenção à saúde. Diretrizes de Atenção à Saúde Ocular na Infância: detecção e intervenção precoce para a prevenção de deficiências visuais. Brasilia: Ministério da Saúde; 2016.

3. World Health Organization (WHO). Statistical Classification of Diseases and Related Health Problems 10th Revision (ICD-10) 2010. [Internet]. [Acessado 2019 jun 24]. Disponível em: http://apps.who.int/classifications/apps/icd/icd10online2005/fr-icd.htm

4. World Health Organization (WHO). Global Data on Visual Impairments 2010. [Internet]. [Acessado 2019 jun 24]. Disponível em: http://www.who. int/blindness/GLOBALDATAFINALforweb.pdf

5. Haddad MAO, Sei M, Sampaio MW, Kara- José N. Causes of visual impairment in children: a study of 3.210 cases. J Pediatr. Ophthalmol Strabismus 2007; 44(4): 232-40.

6. Junior AC, Oliveira LAG. As principais causas de cegueira e baixa visão em escola para deficientes visuais. Rev bras oftalmol. 2016; 75(1):26-29.

7. Haddad MAO, Lobato FJC, Sampaio MW, Kara-José N. Pediatric and adolescent population with visual impairment: study of 385 cases. Clinics. 2006; 61(3):239-46. 
8. Ventura CV, Maia M, Bravo-Filho V, Góis AL, Belfort JR. Zika virus in Brazil and macular atrophy in a child with microcephaly. The Lancet. 2016; 387(10015):228.

9. Verçosa I, Carneiro P, Verçosa R, Girão R, Ribeiro EM, Pessoa A, et al. The visual system in infants with microcephaly related to presumed congenital Zika syndrome. J AAPOS. 2017; 21(4): 300-304.

10. Conselho Brasileiro de Oftalmologia (CBO). As condições de saúde ocular no Brasil. Conselho Brasileiro de Oftalmologia 2019. [Internet]. [Acessado 2019 set 08]. Disponível em: http://www.cbo.com.br/novo/ publicacoes/condicoes_saude_ocular_brasil2019.pdf.

11. Sociedade de Pediatria de São Paulo (SPSP). Recomendações, Atualização de Condutas em Pediatria, gestão 2007-2009. [Internet]. [Acessado 2019 set 08]. Disponível em: https:// www.spsp.org.br/site/asp/recomendacoes/Rec_49_Cegueira.pdf

12. Brito PR, Veitzaman S. Causas de cegueira e baixa visão em crianças. Arq Bras Oftalmol. 2000; 63(1):49-54.

13. Tylefors B. A global initiative for the elimination of avoidable blindness. Am J Ophthalmol. 1998; 125(1):903.

14. Dandona R, Dandona L. Refractive error blindness. Bull World Health Organ. 2001; 79(3):237-43.

15. Resnikoff S, Pascolini D, Mariotti SP, Pokharel GP. Global magnitude of visual impairment caused by uncorrected refractive errors in 2004. Bull World Health Organ. 2008; 86(1): 63-70.
16. Brasil. Ministério da Saúde. Secretaria de atenção à saúde. Diretrizes de Atenção à Saúde Ocular na Infância: detecção e intervenção precoce para a prevenção de deficiências visuais. Brasília: Ministério da Saúde; 2013.

17. Brasil. Ministério da Saúde. Secretaria de atenção à saúde. Diretrizes de estimulação precoce: crianças de zero a 3 anos com atraso no desenvolvimento neuropsicomotor. Brasília: Ministério da Saúde; 2016.

18. Brasil. Portaria no 3.128 , de 24 de dezembro de 2008. Define que as Redes Estaduais de Atenção à Pessoa com Deficiência Visual sejam compostas por ações na atenção básica e Serviços de Reabilitação Visual. Diário Oficial da União. 2008; 24 dez.

19. Colenbrander A. Aspects of vision loss-visual functions and functional vision. Vis Impair Res. 2003; 5(3):115-136.

20. Haddad MA, Siaulys MOC, Sampaio MW. Baixa visão na infância: guia prático de atenção oftalmológica. São Paulo: Laramara; 2011.

21. Oliveira RS, Parizotto AV, Caleffi MF, Beal C, Yeh WSS, Vicensi MC. Avaliação da acuidade visual em escolares no município de Herval d'Oeste, Santa Catarina, Brasil. Rev Bras Med Fam Comunidade. 2013; 8(28):180186.

22. Ferroni MCC. Conhecimento e percepção de escolares com baixa visão sobre sua condição visual, uso de recurso de tecnologia assistiva e expectativas em relação ao futuro [dissertação]. Campinas (SP): Universidade Estadual de Campinas; 2011. 
23. Brasil. Lei $n^{\circ} 9.394$, de 20 de dezembro de 1996. Estabelece as diretrizes e bases da Educação Nacional. Senado Federal. 1996; 20 dez.

24. Ormelezi EM. Aspectos psicossociais da baixa visão na adolescência. In: Sampaio MW, Haddad MAO, Costa Filho HA, Siaulys MOC. Baixa visão e cegueira: os caminhos para a reabilitação, a educação e à inclusão. Rio de Janeiro, RJ: Guanabara Koogan; 2010. P. 373-88.

25. Haddad MAO. Habilitação e Reabilitação Visual de escolares com baixa visão: aspectos médico-sociais [tese]. São Paulo (SP): Universidade de São Paulo; 2006.

26. Corn AL. Visual function: a theorical model for individuals with low vision. J Vis Impairm \& blind. 1993; 77(8):373-77.

27. Brasil. Portaria no 319 , de 26 de fevereiro de 1999. [acessado 2019 ago 13]. Disponível em: http://portal. mec.gov.br/seesp/arquivos/pdf/ port319.pdf

28. Brasil. Lei $n^{\circ} 10.753$, de 30 de outubro de 2003. Institui a Política Nacional do Livro. [acessado 2019 ago 13]. Disponível em: http://www.planalto. gov.br/ccivil_03/Leis/2003/L10.753. htm

29. Brasil. Decreto no 5.296 , de 2 de dezembro de 2004. Regulamenta as Leis nos 10.048, de 8 de novembro de 2000, que dá prioridade de atendimento às pessoas que especifica, $\mathrm{e}$ 10.098, de 19 de dezembro de 2000, que estabelece normas gerais e critérios básicos para a promoção da acessibilidade das pessoas portadoras de deficiência ou com mobilidade reduzida, e dá outras providências. [acessado 2019 ago 13]. Disponível em: http://www.planalto.gov.br/ccivil_03/_Ato20042006/2004/Decreto/D5296.htm
30. Brasil. Decreto $\mathrm{n}^{\mathrm{o}} 7.611$, de 17 de novembro de 2011. Dispõe sobre a educação especial, o atendimento educacional especializado e dá outras providências. [acessado 2019 ago 13]. Disponível em: http://www. planalto.gov.br/ccivil_03/_Ato20112014/2011/Decreto/D7611.ht$\mathrm{m} \# \operatorname{art} 11$

31. Carvalho KMM, Freitas CC, Kimolto EM, Gasparetto MERF. Avaliação e conduta em escolares portadores de visão subnormal atendidos em sala de recursos. Arq Bras Oftalmol. 2002; 65(4):445-49.

32. Brasil. Ministério da Educação. Secretaria de Educação Especial. Sala de recursos multifuncionais: espaços para atendimento educacional especializado. Brasília: MEC/SEESP; 2006.

33. Carvalho KMM, Arruda SMCP, Zimmermann A, Gasparetto MERF, Nobre MIRS, Montilha RCI. Reabilitação: Visão Subnormal e Cegueira. In: Costa MN, Kara-José N. Oftalmologia para o Clínico. Rio de Janeiro: Cultura Médica; 2008. P. 221-34.

34. Carvalho KMM, Gasparetto MERF, Venturini NHB, Kara- José N. Visão subnormal: orientações ao professor do ensino regular. Campinas: Editora da Unicamp; 2005.

35. Brasil. Ministério da Educação Secretaria de Educação Especial Esplanada dos Ministérios. Nota técnica n. 005 / 2011 / MEC / SEESP / GAB. Assunto: Publicação em formato digital acessível - Mecdaisy. [acessado 2019 ago 13]. Disponível em: http://portal.mec.gov.br/index. php?option=com_docman \&view =download \&alias $=9959-$ nota-tec nica-05-2011-secadi\&Itemid $=30192$ 
36. Menezes ET, Santos TH. Verbete CAP (Centro de Apoio Pedagógico para Atendimento às Pessoas com Deficiência Visual). Dicionário Interativo da Educação Brasileira - Educabrasil. São Paulo: Midiamix; 2001. [acessado 2020 fev 07] Disponível em: https://www.educabrasil.com.br/ cap-centro-de-apoio-pedagogico -para-atendimento-as-pessoas-comdeficiencia-visual/

37. Brasil. Decreto $\mathrm{n}^{\circ} 6.286$, de 5 de dezembro de 2007. Institui o Programa Saúde na Escola - PSE, e dá outras providências. Diário Oficial da União. 2007; 6 dez.

38. Malta J, Endriss D, Rached S, Moura T, Ventura L. Desempenho funcional de crianças com deficiência visual, atendidas no departamento de estimulação visual da Fundação Altino Ventura. Arq Bras Oftalmol. 2006; 69(4):571-574.

39. Messa AA, Nakanami CR, Lopes MCB. Qualidade de vida de crianças com deficiência visual atendidas em ambulatório de estimulação visual precoce. Arq Bras Oftalmol. 2012; 75(4):239-242. 